\title{
DIFFERENTIABLE FUNCTIONS ON $c_{0}$
}

\author{
BY JOHN WELLS
}

Communicated by Richard Palais, September 3, 1968.

If $E$ and $F$ are two Banach spaces, denote by $C^{p, q}(E, F), 0 \leqq q$ $\leqq p \leqq \infty$, those functions in $C^{p}(E, F)$ whose derivatives of order less than or equal to $q$ are bounded. Call a Banach space, $E, C^{p, q}$ smooth if there exists a nonzero $C^{p, q}$ function on $E$ with bounded support. Then finite dimensional spaces are $C^{\infty, \infty}$ smooth and if an $L_{p}$ space is $C^{q}$ smooth it is also $C^{q, q}$ smooth. Although $c_{0}$ is known to possess a $C^{\infty}$ (away from zero) norm as described in Bonic and Frampton [1], it is a consequence of the following theorem that $c_{0}$ is not $C^{2,2}$ smooth.

Theorem. Let $f \in C^{1}\left(c_{0}, R\right)$ with $D f$ uniformly continuous. Then the support of $f$ is unbounded.

Proof. If not then there would exist an $f \in C^{1}\left(c_{0}, R\right)$ such that $f(0)=1, f(x)=0$ for $\|x\| \geqq 1$ and $D f$ is uniformly continuous. Pick $N$ such that $\|h\| \leqq 1 / N$ implies $\|D f(x+h)-D f(x)\| \leqq 1 / 2$. Then the mean value theorem gives that $|f(x+h)-f(x)-D f(x)(h)| \leqq 1 / 2\|h\|$ when $\|h\| \leqq 1 / N$. Let $A$ be the set of all $x$ in $c_{0}$ such that $2^{N}-1$ of the first $2^{N}$ components of $x$ have absolute value $1 / N$, the remaining component has absolute value less than or equal to $1 / N$ and all the components after the first $2^{N}$ are zero. Since $A$ is connected and even, we can pick inductively $h_{1}, \cdots h_{N} \in A$ such that $D f\left(h_{1}+\cdots+h_{b-1}\right)$ $\cdot\left(h_{k}\right)=0$ and $h_{1}+\cdots+h_{k}$ has at least $2^{n-k}$ components equal to $k / N$. Then

$$
\left\|h_{1}+\cdots+h_{N}\right\|=1
$$

and

$$
\begin{aligned}
& \left|f\left(h_{1}+\cdots+h_{N}\right)-f(0)\right| \\
& \leqq \sum_{k=1}^{N} \mid f\left(h_{1}+\cdots+h_{k}\right)-f\left(h_{1}+\cdots+h_{k-1}\right) \\
& \quad-D f\left(h_{1}+\cdots+h_{k-1}\right) h_{k} \mid \leqq \sum_{k=1}^{N} \frac{1}{2}\left\|h_{k}\right\|=\frac{1}{2}
\end{aligned}
$$

which is a contradiction.

Corollary 1. Let $f \in C^{1}\left(c_{0}, R\right)$ and $D f$ be uniformly continuous. Then $f(\delta U)$ is dense in $f(U)$ for all bounded open sets $U$. 
COROLlary 2. There exists a closed subset of $c_{0}$ which is not the loci of zeros of a $C^{2}$ function.

Proof. Consider the complement of a sequence of disjoint open balls converging to a point.

The $C^{\infty}$ norm described in [1] has first derivative bounded by one and by composing with a suitable function in $C^{\infty}(R, R)$ we get a $C^{\infty, 1}$ function on $c_{0}$ with bounded support. The following is another example of a $C^{\infty, 1}$ function.

Let $\eta \in C^{\infty}(R, R), \eta(t) \geqq 0, \eta(t)=0$ if $|t| \geqq 1 / 4$ and $\int_{-1 / 4}^{1 / 4} \eta(t) d t=1$. Define

$$
\begin{aligned}
\phi_{n}(x)= & \int_{-1 / 4}^{1 / 4} \cdots \int_{-1 / 4}^{1 / 4} \eta\left(y_{1}\right) \cdots \eta\left(y_{n}\right) \\
& \cdot F\left(x_{1}+y_{1}, \cdots, x_{n}+y_{n}, x_{n+1}, \cdots\right) d y_{1} \cdots d y_{n}
\end{aligned}
$$

where $F(x)=\inf _{\|y\| \leq 1}(\|x-y\|), x=\left\{x_{1}, x_{2}, \cdots\right\}, y=\left\{y_{1}, y_{2}, \cdots\right\}$. Suppose that $\left|x_{m}\right| \leqq 1 / 4$ if $m>n(x)$. Now if $\left\|x^{\prime}-x\right\| \leqq 1 / 4,\|y\| \leqq 1 / 4$ and $x_{m}^{\prime}=x_{m}$ for $m \leqq n(x)$, then $F\left(x^{\prime}+y\right)=F(x+y)$. Hence when $\|z-x\| \leqq 1 / 4, \phi_{n(x)}(z)$ depends only on the first $n(x)$ coordinates and therefore is $C^{\infty}$. Also $\left\|x^{\prime}-x\right\| \leqq 1 / 4,\|y\| \leqq 1 / 4$ and $y_{1}=\cdots=y_{n(x)}=0$ imply that $F\left(x^{\prime}+y\right)=F\left(x^{\prime}\right)$. Hence $\phi_{m}\left(x^{\prime}\right)=\phi_{n(x)}\left(x^{\prime}\right)$ when $m \geqq n(x)$ and $\left\|x^{\prime}-x\right\| \leqq 1 / 4$. The above implies that $\phi(x)=\lim _{n \rightarrow \infty} \phi_{n}(x)$ exists and is $C^{\infty}$ for all $x$. Now $\left|\phi_{n}(x)-\phi_{n}(z)\right| \leqq \int_{-1 / 4}^{1 / 4} \cdots \int_{-1 / 4}^{1 / 4} \eta\left(y_{1}\right) \cdots$ $\eta\left(y_{n}\right)\|x-z\| d y_{1} \cdots d y_{n}=\|x-z\|$. Hence $|\phi(x)-\phi(z)| \leqq\|x-z\|$ which gives $\|D \phi(x)\| \leqq 1$ for all $x$. Finally let $\rho \in C^{\infty}(R, R), 0 \leqq \rho \leqq 1, \rho(t)=1$ if $t \leqq 0$ and $\rho(t)=0$ if $t \geqq 3 / 4$. Then $\rho(\phi(x)) \in C^{\infty, 1}\left(c_{0}, R\right), \rho(\phi(0))=1$ and the support of $\rho(\phi(x))$ is contained in the unit ball.

\section{BIBLIOGRAPHY}

1. R. Bonic and J. Frampton, Smooth functions on Banach manifolds, J. Math. Mech. 15 (1966), 877-898.

California Institute of Technology, Pasadena, California 91109 